discharge axis repels incoming electrons, which are driven away from the axis by the resulting electric field. Xenon excitation is, however, smaller at $t=169 \mathrm{~ns}$ than at $t=$ 159.7 ns (i.e., the centres of the excited regions appear in a less intense red colour owing to the significant decrease in the discharge current).

\section{Research Issues}

A weakly ionized plasma represents a complex system in which electrons, positive ions, excited species, and photons interact together and with the electric field. A better qualitative and quantitative understanding of the physical phenomena occurring in a PDP cell is being achieved by developing sophisticated, user-friendly numerical models.

The luminous efficiency of PDPs has been considerably increased in the last few years, but it is still relatively low (on the order of $1 \mathrm{~lm} / \mathrm{W}$ ) and one of the issues in PDP technology is the improvement of this parameter. The low efficiency is partly due to the fact that a large proportion of the electrical energy is dissipated by positive ions in the high-field sheath region, through collisions with neutral atoms or with the walls. Improvement of the luminous efficiency can be achieved by improving the cell's geometrical design, optimizing the gas mixture and using phosphors with a higher quantum efficiency. Lowering the operating voltage is also an important issue, and could be achieved by increasing the secondary electron emission of the film covering the dielectric layers and the gas mixture. This is mainly a cost issue since the technology of high-voltage $(200 \mathrm{~V})$ integrated driver circuits is now under control.

Addressing speed is another important issue because it impacts on the greyscale rendition, and more generally on image quality (moving picture rendition). Improvements in this area require action both on the physics of the PDP (time response of the cells) and on the electronic driving circuit.

These research issues invariably require a better understanding of the physical mechanisms involved in plasma display panels, so sophisticated but userfriendly numerical modelling of the discharge cells represents a way to achieve substantial technical improvements.

The Panilevé Property, A Century Later School - Cargèse, France; 3-22 June 1996

Contact: R. Conte, DRECAM-SPEC, CE Saclay, F-91191 Gif-sur-Yvette - E-mail: conte@amoco.saclay.cea.fr Tel.: +33-1- 69087349 - Fax: +33-1-69088786

\title{
Painlevé a Century Later
}

The signal which propagates along a typical optical fibre used for trans-oceanic communication comprises a solitary wave, with an exact analytic expression depending on very few adjustable parameters. Tremendous effort is needed to amplify the signal every $400 \mathrm{~km}$. Present theory predicts the probable existence of an exact, essentially attenuation-free solution with more free parameters. Its discovery, a difficult task, will necessarily involve methods for integrating nonlinear differential equations that were articulated a century ago by the French mathematician Paul Painlevé, whose contributions will be marked by a Cargèse school this spring. In covering both the old, but not obsolete, methods as well as modern extensions to physics, it will be honoured by the presence of Professor Bureau, the last disciple of the Painlevé school, and by Professor Kruskal, the co-discoverer of the soliton.

The Painlevé property states that in order to define a function, a differential equation must have a general solution without any branching around the singular points whose positions depend on the initial conditions. All linear equations have this property and provide the elements for building analytic expressions (polynomials, exponentials, trigonometric, Bessel, etc., functions). Nonlinear equations, on the other hand, provide very few, new functions. For instance, first order only defines one new function, the elliptic function (the famous inverse function $u(x)$ of the so-called elliptic integral $x(u)$ ruling the motion of the pendulum).

It was the systematic examination of all differential equations of second order which in 1898-1906 led Painlevé and his student Gambier to the discovery of six new functions defined by a differential equation, the first - $\mathrm{P}_{1}$ - being simply $d^{2} u / d x^{2}=6 u^{2}+x$. Painlevés feat for long only excited the curiosity of a few mathematicians, since the fashion turned to more abstract mathematics.

The renewal of interest dates backs to the end of the 1960 s, pushed by three apparently distinct domains of physics. First, the discovery of the soliton in 1965 initiated the booming domain of integrable evolution equations, i.e., those admitting elastic collisions of quasiparticles called multi-solitons [1]. The link with Painlevé is that, whenever such an integrable equation admits what is technically called a noncharacteristic reduction to an ordinary differential equation (ODE), this ODE has the Painlevé property. One example is the Korteweg-de Vries equation for $U(X, T)$,

$$
U_{T}+U_{X X X}-12 U U_{X}=0,
$$

and its reduction to ( $\left.\mathrm{P}_{1}\right)$ for $u(x)$,

$$
u(x)=U(X, T)+T ; x=X-6 T^{2} .
$$

Second, statistical physics and field theory have provided a number of exactly solvable models, in which typically the free energy or some correlation function, as a function of say the temperature, obeys a Painlevé equation. A celebrated example is the two-dimensional Ising model while the self-dual Yang-Mills equations, fundamental to field theory, admit a reduction to the master Painlevé equation P6.

Third, the advent of computers has popularized the observation that dynamic systems such as the Lorenz model of atmospheric circulation,

$$
x^{\prime}=\sigma(y-x) ; y^{\prime}=-x z+r x-y ; z^{\prime}=x y-b z
$$

although deterministic, i.e., first order in time, generically exhibit chaotic behaviour. However, for some values of the control parameters $(b, \sigma, r)$, easily found by the so-called Painlevé test, they may have the Painlevé property. For instance, the case of $b=0, \sigma=1 / 3$ is integrable with $\mathrm{P}_{3}$.

The renewal in interest has produced feedback in two major directions: the extension of the theory of Painlevé to partial differential equations [2] and, more recently, to finite difference equations [3] under the impulse of statistical lattice physics. In our youth we learnt many recipes for integrating nonlinear differential equations. But simple and powerful methods to achieve the goal when integration is possible were developed a century ago in Painlevé's famous Leçons de Stockholm [4]. This text, although temporarily - one hopes - out of print, remains a reference.

R. Conte, CE Saclay

References,

[1] Ablowitz M.J., Clarkson P.A., Solitons, nonlinear evolution equations and inverse scattering, LMS Lecture Note Series (Cambridge University Press, 1991). [2] Weiss J., Tabor M. \& Carnevale G., J. Math. Phys. 24 (1983) 522 .

[3] Brézin E., Kazakov V.A., Phys. Lett. B 236 (1990) 144; Gross D.J., Migdal A.A., Phys. Rev. Lett. 64 (1990) 127.

[4] Oeuvres de Paul Painlevé, 3 vols. (Editions du CNRS, Paris; 1973, 1974, 1976). 